\title{
Segmented Aperture Interferometric Nulling Testbed (SAINT) II: component systems update
}

Brian A. Hicks, Matthew R. Bolcar, Michael A. Helmbrecht, Peter Petrone, Elliot Burke, et al.

Brian A. Hicks, Matthew R. Bolcar, Michael A. Helmbrecht, Peter Petrone, Elliot Burke, James Corsetti, Thomas Dillon, Andrew Lea, Samuel Pellicori, Teresa Sheets, Ron Shiri, Jack Agolli, John DeVries, Andrew Eberhardt, Tyler McCabe, "Segmented Aperture Interferometric Nulling Testbed (SAINT) II: component systems update," Proc. SPIE 10400, Techniques and Instrumentation for Detection of Exoplanets VIII, 104001I (1 September 2017); doi: $10.1117 / 12.2273905$

Event: SPIE Optical Engineering + Applications, 2017, San Diego, California, United States 


\title{
The Segmented Aperture Interferometric Nulling Testbed (SAINT) II: component systems update
}

\author{
Brian A. Hicks ${ }^{*} a, b$, Matthew R. Bolcar ${ }^{b}$, Michael A. Helmbrecht ${ }^{c}$, Peter Petrone III ${ }^{d, b}$, \\ Elliot Burke ${ }^{e}$, James Corsettif ${ }^{f, b}$, Thomas Dillon ${ }^{g}$, Andrew Lea ${ }^{h, b}$, Samuel Pellicori ${ }^{i}$, \\ Teresa Sheets $^{b}$, Ron Shiri ${ }^{b}$, Jack Agolli $^{j}$, John DeVries ${ }^{k}$, Andrew Eberhardt ${ }^{l}$, Tyler McCabe $^{m}$ \\ ${ }^{a}$ University of Maryland, College Park, MD, USA \\ ${ }^{b}$ NASA Goddard Space Flight Center, Greenbelt, MD, USA \\ ${ }^{c}$ Iris AO, Inc., Berkeley, CA, USA \\ ${ }^{d}$ Sigma Space Corporation, Lanham, MD, USA \\ ${ }^{e}$ HighTide Instruments, Oak View, CA, USA \\ fUniversity of Rochester, Rochester, NY, USA \\ ${ }^{g}$ University of Delaware, Newark, DE, USA \\ ${ }^{h}$ Stinger Ghaffarian Technologies, Inc., Greenbelt, MD, USA \\ ${ }^{i}$ Pellicori Optical Consulting, Santa Barbara, CA, USA \\ ${ }^{j}$ Worcester Polytechnic Institute, Worcester, MA, USA \\ ${ }^{k}$ University of Illinois, Urbana-Champaign, IL, USA \\ ${ }^{l}$ University of Washington, Seattle, WA, USA \\ ${ }^{m}$ Florida Institute of Technology, Melbourne, FL, USA
}

\begin{abstract}
This work presents updates to the coronagraph and telescope components of the Segmented Aperture Interferometric Nulling Testbed (SAINT). The project pairs an actively-controlled macro-scale segmented mirror with the Visible Nulling Coronagraph (VNC) towards demonstrating capabilities for the future space observatories needed to directly detect and characterize a significant sample of Earth-sized worlds around nearby stars in the quest for identifying those which may be habitable and possibly harbor life. Efforts to improve the VNC wavefront control optics and mechanisms towards repeating narrowband results are described. A narrative is provided for the design of new optical components aimed at enabling broadband performance. Initial work with the hardware and software interface for controlling the segmented telescope mirror is also presented.
\end{abstract}

Keywords: Exoplanets, high-contrast imaging, nulling interferometry, wavefront sensing and control, space telescopes, segmented mirrors

\section{PROJECT SCOPE}

In support of NASA's Exoplanet Exploration Program and the Technology Demonstration for Exoplanet Missions (TDEM) component of NASA's Strategic Astrophysics Technology (SAT) program, the Visible Nulling Coronagraph (VNC) at NASA's Goddard Space Flight Center is being coupled with an actively-controlled macro scale segmented primary mirror via a fine pointing system (FPS) to form the Segmented Aperture Interferometric Nulling Testbed (SAINT). SAINT is being developed as an end-to-end coronagraphic system to demonstrate high-contrast imaging in the presence of complex diffraction and environmental instabilities with traceability to future large space telescopes having coronagraphic high-contrast imaging capability. The project's final milestone objective comprises holding a contrast of $10^{-8}$ at an inner working angle (IWA) of $4 \lambda / D$ within a bandpass of $\Delta \lambda=20 \mathrm{~nm}$ centered near $633 \mathrm{~nm}$ for 1,000 seconds on three separate occasions, with a goal of $10^{-9}$ at an IWA of $3 \lambda / D$ over a $\Delta \lambda=40 \mathrm{~nm}$ bandpass. Intermediate milestone goals include reproducing or exceeding earlier TDEM narrowband performance ${ }^{1,2}$ and achieving for the first time a broadband high-contrast demonstration

\footnotetext{
*bahicksmail@gmail.com
}

Techniques and Instrumentation for Detection of Exoplanets VIII, edited by Stuart Shaklan, Proc. of SPIE Vol. 10400, 104001I · @ 2017 SPIE · CCC code: 0277-786X/17/\$18 · doi: 10.1117/12.2273905 

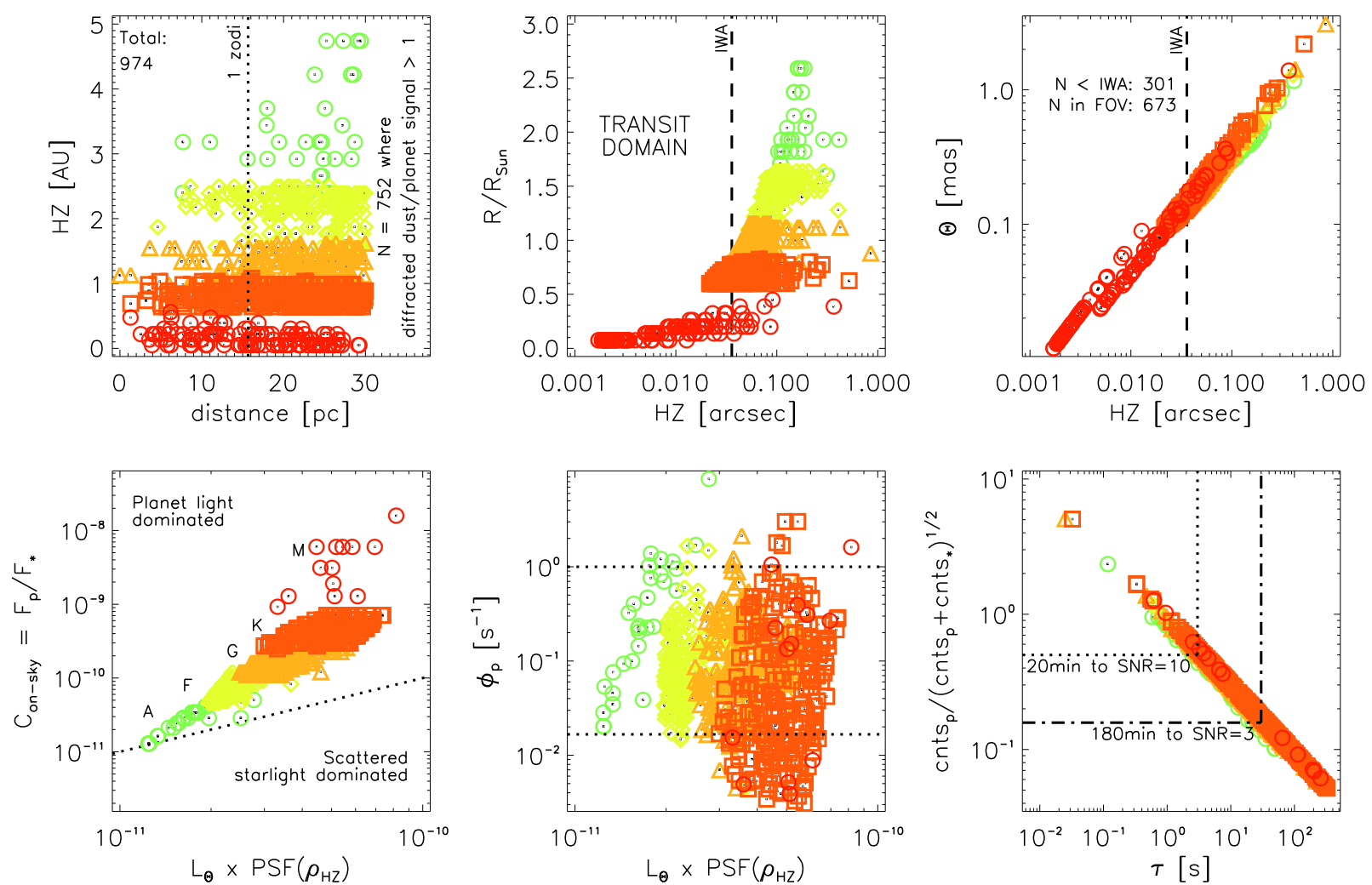

Figure 1. Accessibility of hypothetical Earth-like planets in HZ orbits around A, F, G, K, and M stars within 30pc of Earth based on system resolution, contrast, and photometric capability. R-band fluxes and diffraction are calculated assuming a $15.1 \mathrm{~m}$ 6-ring hexagonal array primary mirror architecture paired with a dual $D / 13$ shearing VNC yielding $\sim 9 \%$ total throughput including optical transmission losses and detector quantum efficiency, an IWA of $4.1 \lambda / D$, and a point spread function scattering roll-off profile following $\mathrm{PSF} \propto \rho^{-2.5}$. Approximately $10-20$ of all the systems plotted will host transiting planets. Further description of the plots shown in this figure is provided in the text.

with the VNC alone. Satisfying these TDEM milestone objectives are the next steps in establishing the VNC as a viable option for a future large space telescope requiring capability for directly detecting and characterizing Earth-like exoplanets.

Identifying and characterizing potentially habitable planets around nearby stars are motivating science goals for future space telescopes. The telescopes will need to be large to address exoplanet direct imaging resolution and photometric flux constraints and may likely build upon hexagonal array segmented mirror infrastructure in order to fit inside of limited launch fairing volumes. Sensitivity to any existing Earth-like planets orbiting the many hundreds of nearby stars may be achieved by pairing an adequately-sized telescope with a coronagraphic starlight suppression system. The plots shown in the top row of Fig. 1 present key physical parameters of nearby stellar systems including distances, stellar types and sizes $\left(R / R_{S u n}\right)$, mean habitable zone (HZ) orbital separations, and corresponding calculated characteristic stellar angular diameters $(\Theta)$ and orbital angular separations. Vertical lines are plotted indicating the 1 zodi signal parity distance ${ }^{3}$ assuming R-band observations with a $15.1 \mathrm{~m}$ telescope, and a $4.1 \lambda / D$ IWA.

The bottom row of plots in Fig. 1 estimate system on-sky contrast $\left(F_{p} / F_{*}\right)$ and planet reflected light photon count rates $\left(\phi_{p}\right)$, time constants for counting a single reflected light photon $\left(\tau=1 / \phi_{p}\right)$, and noiseless detector signal-to-noise ratio (SNR) for one second exposures assuming planets are observed at mean HZ distance from their host stars at half phase with sizes and albedos identical to Earth. The high-contrast system assumes the above telescope and an R-band optimized VNC that "leaks," diffracts, and scatters starlight in proportion to $L_{\Theta} \times P S F \propto \Theta^{4} \rho^{-2.5}$ where $\rho$ is the radial off-axis separation, here assumed to be the projected mean $\mathrm{HZ}$ 

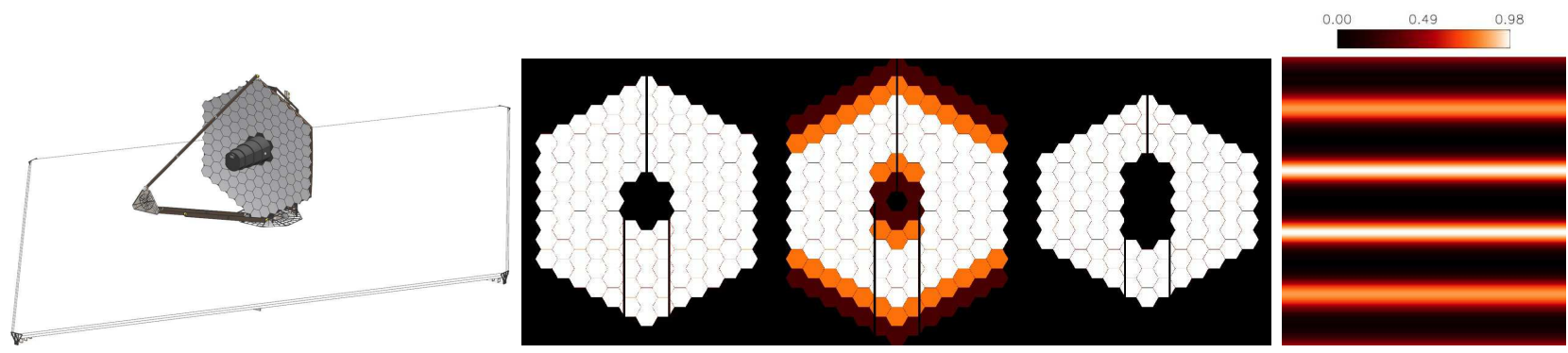

Figure 2. The principle of operation of a dual lateral shear VNC paired with a large 6-ring hexagonal aperture segmented telescope. Individual shears by a single "rank" of segments are imparted inside of sequential beamsplitter pairs that define each interferometer cavity. The rightmost panel shows a corresponding $20 \%$ bandpass interferometric transmission plot that is $\approx 40 \lambda / D$ on a side. Neglecting detector quantum efficiency and polarization losses, additional transmission losses associated with beamsplitter efficiency, multiple mirror reflections, and aperture masking reduce transmission reduce constructive fringe peak transmission to $12 \%$.

distance from the star. As shown in the lower right plot, for a select few systems, it could be possible to acquire modest SNR spectra in reasonable amounts of time for Earth-like planets in HZ orbits, should they be detected around the nearest stars.

It is important to recall that, as a lateral shearing nulling interferometer, the VNC places a periodic transmission pattern on the sky, which is depicted for an assumed 6-ring segmented aperture in Fig. 2. In its most simplistic form, the VNC works by destructive and constructive interference: placing a star on the destructive interference central minimum (null) moves the stars light from the "dark" high contrast output to a "bright" camera observing the pupil for wavefront control. Conversely, placing the planet on a constructive interference maximum allows transmission of the planet light. While other important scaling laws pertaining to phase, amplitude, and chromatic errors are presented in greater detail in earlier works and references therein, $, 1,2,4,5$ the transmission function resulting from the coherent combination of electric fields through $j$ sequential nuller cavities is given by the product

$$
E_{t} \propto \prod_{j=1}^{N_{s}}\left(1-e^{\frac{2 \pi \mathbf{r} \cdot \mathbf{s}_{j} i D}{\lambda}}\right)
$$

where $\mathbf{r}$ and $\mathbf{s}_{\mathbf{j}}$ are the normalized pupil coordinate and fractional shear vectors, respectively. Taking the complex square of this function for an arbitrary number of shears, the ideal (wavefront error free) transmission pattern at a single wavelength is given by

$$
T(x, y)=\prod_{j=1}^{N_{s}} \sin ^{2} \frac{\pi D \mathbf{r} \cdot \mathbf{s}_{j}}{\lambda}
$$

Typically, for visible nulling with telescopes having aperture diameters on the order of $1 \mathrm{~m}$ to $10 \mathrm{~m}$, dual fractional shears of $\mathrm{D} / 4$ or smaller adequately limit the transmission of the finite angular extent of a star while preserving off-axis transmission. Greater shears reduce the IWA at the cost of reduced throughput and increased sensitivity to stellar angular extent and pointing jitter. Shearing twice in the same direction (the y-direction in Fig. 2) by a single rank of hexagonal segments for a 6-ring hexagonal array yields a transmission function of

$$
T(x, y)=\sin ^{4} \frac{\pi D y}{13 \lambda}
$$

Given the periodic nature of the transmission pattern for the VNC in particular, and the importance to an arbitrary coronagraph's observation campaign strategy in general, we note that the contrast and count rates presented in Fig. 1 will vary significantly depending on the epoch of observation for a given system. While some a priori knowledge of planetary orbital parameters may be provided by indirect detection methods, it may be 
expected that a future direct imaging discovery search campaign will largely observe planets at random on-sky positions and phases. Under these realistic circumstances, system contrast, separation, and position angle were modeled using Monte Carlo methods for hypothetical Earth-like planets in random HZ orbits, and these effects are presented in Fig. 3. The modeled random HZ orbits assumed bounds on orbital eccentrencity that were calculated using methods similar to those in a related work. ${ }^{6}$ Given higher contrast as a function of phase or the chance of a HZ planet falling outside the range bounded by the coronagraphic IWA and outer working angle (OWA), follow-up observations for non-detections around the nearest mature F, G and K-type systems of interest should be carefully planned for not just a single mission, but also taking into account more distant future missions that may drastically improve the efficiency of detection. As stated in Stark, et al. (2014), "proper calculation of how revisits impact mission yield requires optimizing the time between visits." 7 Expanding on this remark, it should be possible to further optimize the economy of impending missions by developing prioritization strategies for detection and characterization based on statistical modeling spanning into future missions.
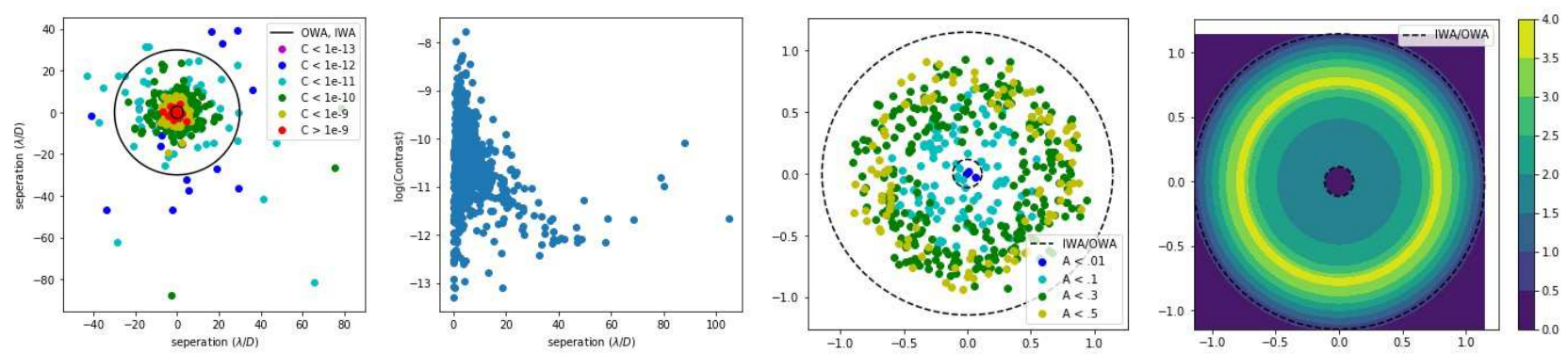

Figure 3. Left plots: on-sky projected planet locations and contrasts and the corresponding angular separation and contrast scattergram assuming randomized HZ orbits around stars within 30pc. Right plots: randomized projected locations and resultant phase fraction at each location, and the probability distribution function of a HZ planet orbiting $\tau$ Ceti with the spatial scale normalized to mean HZ semi-major axis. All plots assume R-band observations with a $15.1 \mathrm{~m}$ telescope with $4 \lambda / D$ IWA and $30 \lambda / D$ OWA.

\section{SYSTEM AND CONTROLS OVERVIEW}

The SAINT system pairs an actively-controlled segmented telescope operating in an enclosure within an ambient laboratory environment with the VNC, which operates inside a vacuum chamber. The chamber provides an isolated environment for demonstrations at atmospheric pressure, at reduced pressure similar to a stratospheric balloon environment, or at high vacuum to emulate system operation in a space-like environment. The vacuum chamber rests atop a $16 \times 4$ stabilized air table that supports the full system. Inside the chamber, the VNC breadboard rests on a shelf on additional passive isolators. Vacuum feedthroughs consisting of electrical cables

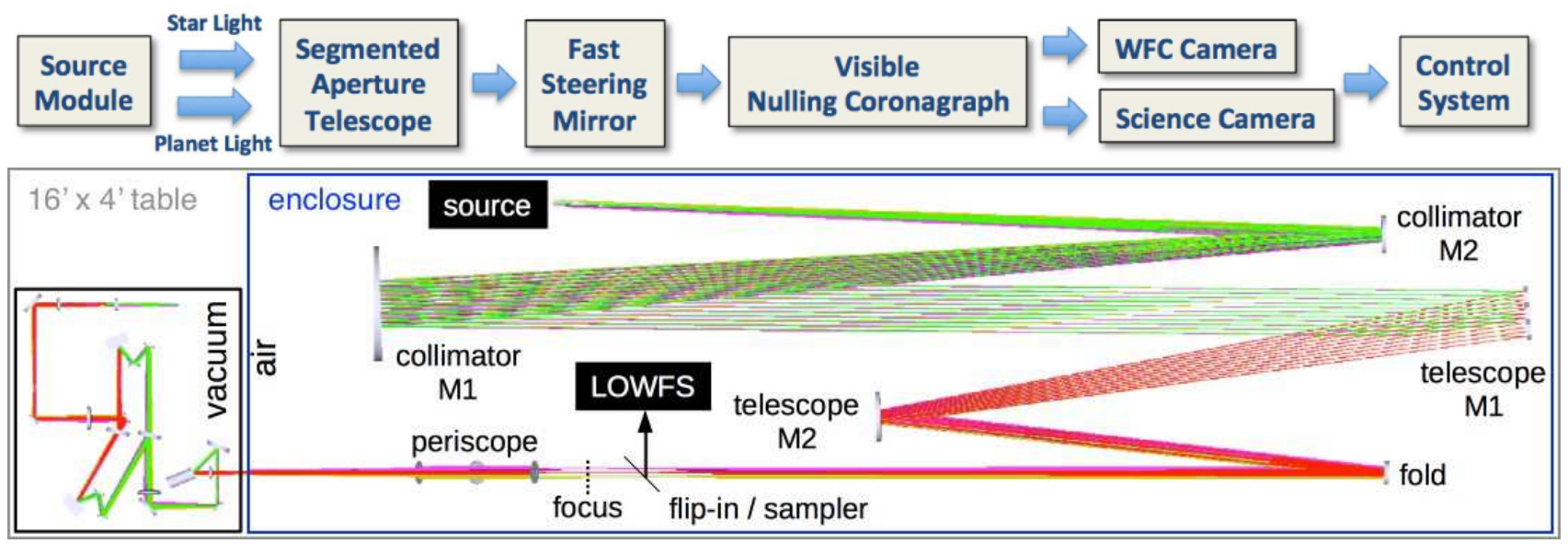

Figure 4. The SAINT top-level end-to-end tiered active control system block diagram and optical layout. 


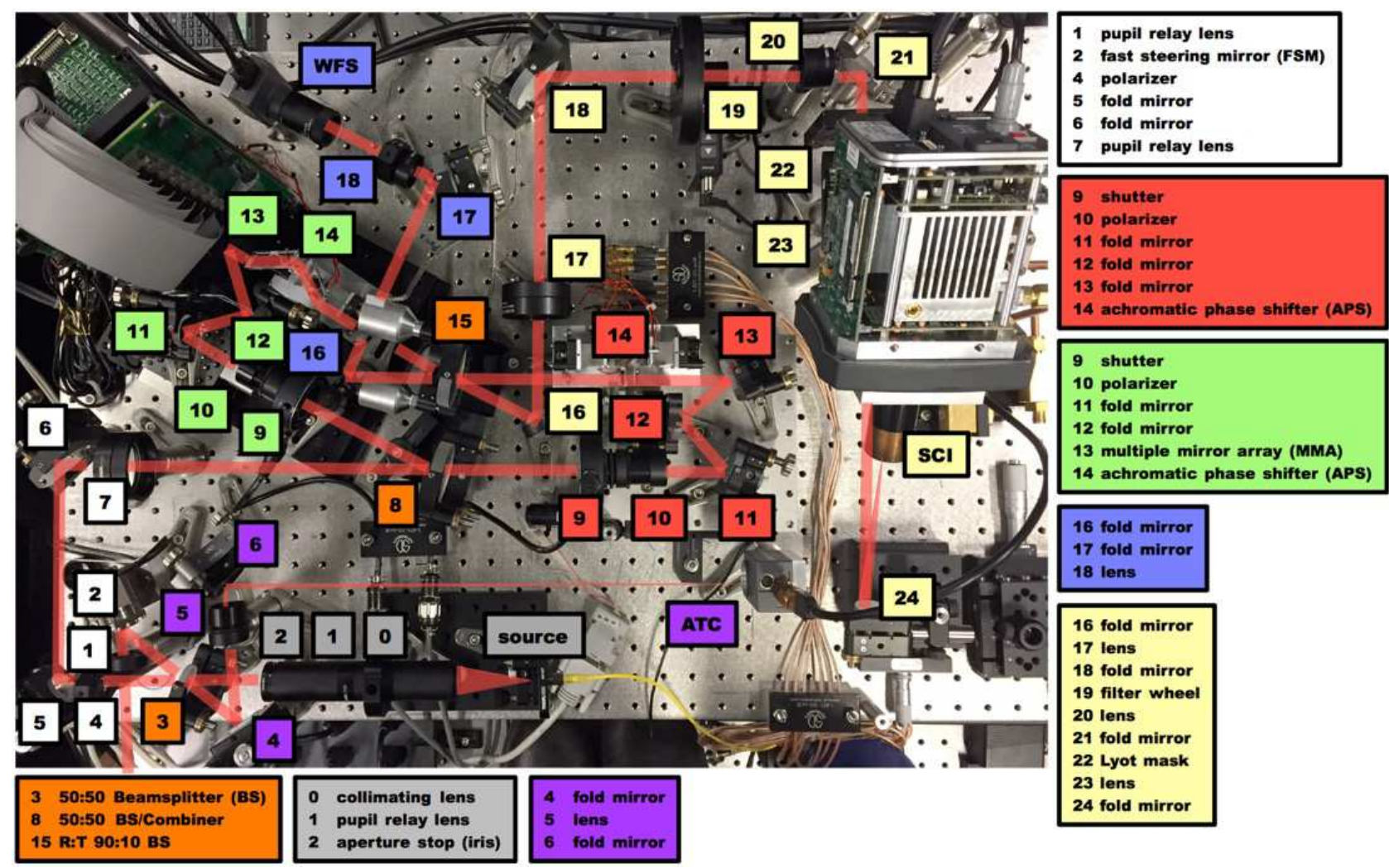

Figure 5. The VNC breadboard as of fall 2016 including: a compacted source module [grey], a fully active Iris AO PTT489 segmented DM (a.k.a. multiple mirror array) [green], an upgraded delay arm [red] stage with 0.5nm closed-loop absolute positioning - previously $\sim 1 \mu \mathrm{m}$, the FPS optics [purple] to couple the VNC to the active segmented telescope, modifications to aft-optics [yellow] replacing adjustable zoom lenses with fixed focus achromats, as well as bandpass filters moved to the destructive "dark" high-contrast output to allow constant tracking of the full source bandpass central broadband constructive fringe with the "bright" output wavefront sensor (WFS) optics [blue].

and water chiller lines to cool the high-contrast focal plane detector pass through multiple tank bulkheads. Fig. 4 shows a layout of the end-to-end optical system. The telescope optics form one of two selectable source inputs for the VNC, the other being a circular aperture with Gaussian intensity profile for intermediate milestone narrowband and broadband demonstrations of the $\mathrm{VNC}$ alone.

Fig. 5 shows the VNC including the addition of the FPS. The VNC uses a combination of active and passive components to achieve the desired contrast at small IWA over a finite spectral bandpass. The beamsplitter/combiner (BS/C) separate then recombine the two "arms" of the interferometer. A segmented Iris AO PTT489 deformable mirror (DM - labeled "multiple mirror array" in Fig. 5) corrects high-spatial frequency noncommon path errors relative to the reference delay arm used to minimize piston optical path difference. Shutters and linear polarizers in each arm are used to measure and balance amplitude, respectively. Aft-optics relay the bright and dark output beams to wavefront sensing and science cameras, respectively.

The computer (CPU) interface and software control development for the full system is presently distributed between three computers, all running the same Linux OS. The first CPU interfaces the SAINT M1 low-order wavefront sensor (LOWFS) detector \& wavefront control (WFC) in the form of rigid body piston/tip/tilt (PTT) actuation of segments via 3-axis piezoelectric transducer ("piezo" or PZT) mounts. The second CPU interfaces the FPS fast steering mirror (FSM) amplifiers for the tip-tilt PZT stage and readout for strain gauge sensors, providing closed-loop displacement feedback for each axis, as well as an angle tracker camera (ATC) to close the loop on stabilizing pointing jitter. The third CPU commands and reads out components of the VNC including the DM, shutters, delay stage, and achromatic phase shifter (APS) positioners, the "dark" channel high-contrast science detector (SCI), and the high-order "bright" channel WFS. 

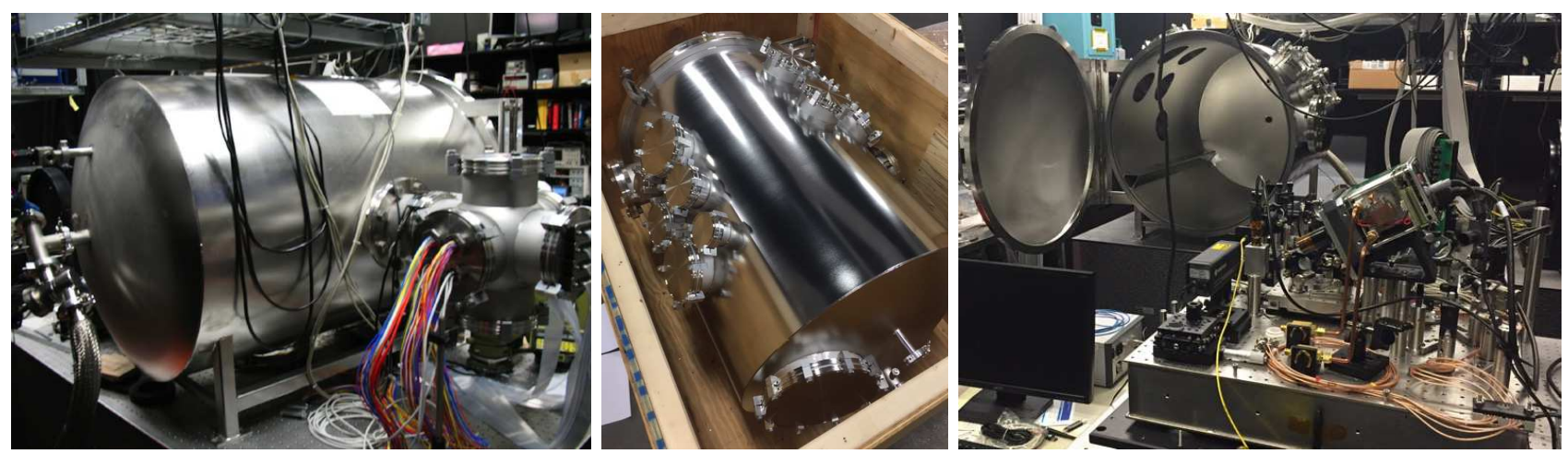

Figure 6. Left: the VNT chamber as used in TDEM-10 (MS\#2). Center: the modified chamber received on 9/20/16. Right: the upgraded VNT chamber following successful testing on 9/21/16 with a combination of port blanks and previously tested feedthroughs, ready to be used toward meeting TDEM-13 Milestones \#1.5, \#2.5, and \#3.

\section{DEVELOPMENT NARRATIVE}

SAINT development builds on the VNC TDEM Milestone \#1 demonstration of $<10^{-8}$ contrast at an IWA of $2 \lambda / D$ in narrowband light, ${ }^{1,2}$ and the Milestone \#2 effort to increase contrast and spectral bandpass through the inclusion of achromatic phase shifters (APS). ${ }^{8-11}$ New air-side system additions including a source collimator, telescope, periscope, and LOWFS feed optics have all been integrated and aligned, and the M1 segments have been coarse aligned to fall within the range of fine control rigid body PTT piezo actuators. ${ }^{12}$ The remainder of this work presents updates on addressing a number of additional experiment needs identified in the VNC TDEM-10 Milestone \#2 report $^{11}$ and TDEM-13 Milestone \#3 whitepaper. $^{13}$

Modifications to the VNC breadboard described in Sec. 2 above began in late summer 2016. Fore-optics were redesigned to enable coupling to the air-side segmented telescope and include the FSM and ATC to form the FPS for pointing offset correction and jitter compensation. The aft-optics were simplified to address alignment drift and chromatic aberrations previously observed on the dark channel science camera. Concurrent with these redesign and rebuild efforts, long lead time, pressingly needed hardware items were prioritized, improved performance designs for broadband optics were (and are still being explored - see Sec. 4), access and feedthrough ports to the VNC vacuum chamber were added (see Fig. 6), and a high-precision, high-accuracy long travel delay stage was acquired and integrated (see Fig. 7).

The decision to invest in adding ports to the chamber addressed working with a cumbersome six-way cross shown in the left photo of Fig. 6 that presented a cabling challenge when moving the VNC in and out of its chamber, adding significant risk that costly high-density signal leads would be damaged, or sometimes inadvertently introducing a misalignment requiring repeating alignment procedures and checks external to the chamber. A total of eleven ports were added to accommodate the growing number of electrical feedthroughs including a larger format DM, as well as one adding working access through the rear of the chamber visible in the central photo of Fig. 6. The chamber has been in a ready and tested state since late 2016.
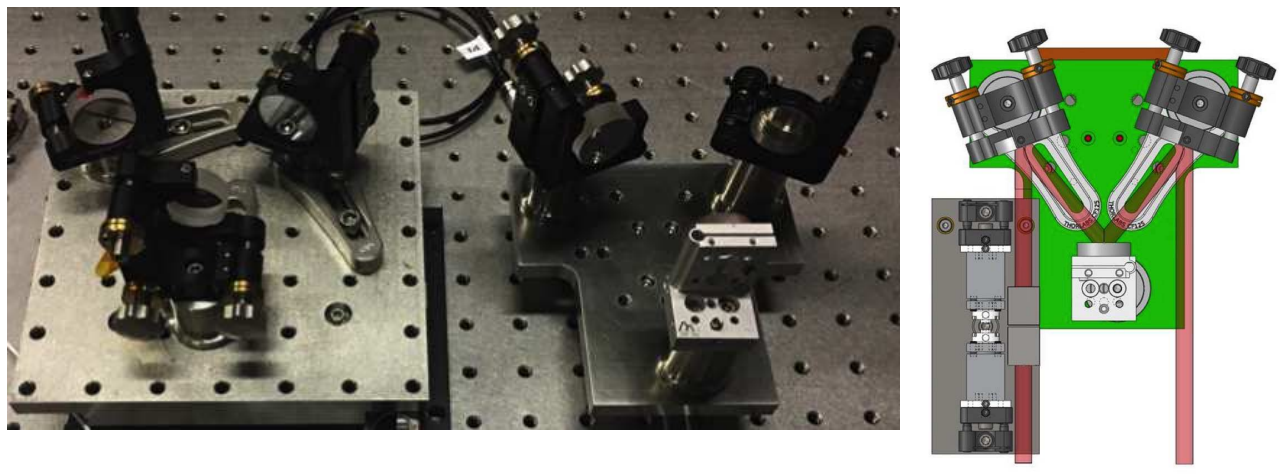

Figure 7. A previous generation delay stage (photo left) with long travel stepper motor and sub-nm resolution PZT was replaced by a Physik Instrumente Piezowalk device (photo right) to achieve the same range, but with 100-1000x better absolute coarse positioning enabled by an encoder and servo control. 

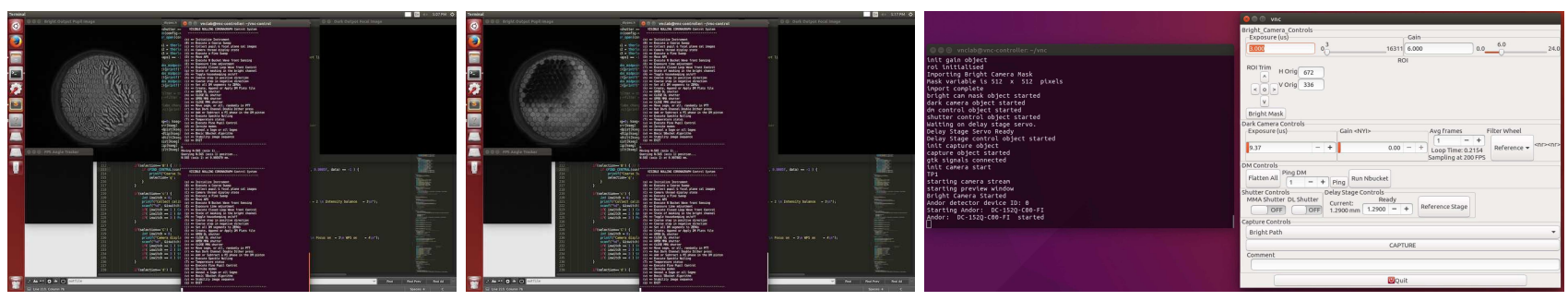

Figure 8. Left: a demonstration of flattening a fully active Iris AO PTT489 with the VNC using the updated Linux OS first performed on January 26, 2017 using the upgraded VNC. Right: The GUI being developed to simplify control of detector settings and perform a more diverse range of routine alignment, phasing, and calibration routines.

Related to the alignment drifts and challenges of needing to perform alignment and alignment checks outside the chamber, a hybrid coarse stepper and fine piezo delay stage used to remove piston optical path difference between nuller arms was replaced with a new delay stage enabling $0.5 \mathrm{~nm}$ closed-loop absolute positioning and stability over a $10 \mathrm{~mm}$ range (see Fig. 7). Performing this upgrade removed a high voltage amplifier and addressed backlash on the order of 1-2 $\mu \mathrm{m}$ observed with the previous stage. While the stroke of the piezo used for fine actuation was adequate to compensate for absolute errors related to the backlash, slow creep observed following coarse actuation necessitated a wait period before fine control high-contrast demonstrations could be attempted. An anomalous resonance under load observed in the initial closed-loop operational tests of the upgraded stage was addressed through firmware, and device control was demonstrated using newly written software routines by the end of calendar year 2016.

Writing code to integrate control of the new delay stage was part of a greater VNC system and software migration to an up-to-date OS running on a faster CPU that began in the late summer of 2016. Significant work has been completed on the VNC control CPU to parse and restructure the code used in earlier demonstration. An initialization file outlines the detector exposure settings, DM configuration files, delay line initial position and rate of motion, as well as filter settings. At the time of writing this manuscript, all of the automated closed-loop wavefront sensing and control routines remain to be tested on the new OS. Control of a full-yield Iris AO PTT489 was demonstrated using the updated control CPU in January 2017 (see Fig. 8). The demonstration used the upgraded VNC, including the addition of the FPS optics that were instlled, but not used, and the new high-accuracy delay stage. Concurrent with developing a detector multithreading framework, a graphical user interface (GUI) has been developed to adapt control of the VNC to a wider user base. A screenshot of the GUI is shown on the right of Fig. 8.

Other new devices requiring updated drivers and application program interface and software development kits included the ATC and FSM components of the FPS, a new CMOS WFS compliant with the up-to-date OS, and a motorized filter wheel. In addition to the VNC code migration, new basic handles were programmed for controlling the SAINT M1 and FPS shown in Fig. 9 and Fig. 10, respectively. While the VNC controls are largely in place and ready to be tested with heritage algorithms, significant work remains to be completed to achieve automated alignment of the SAINT M1 and close the loop on pointing stabilization. Command line interface
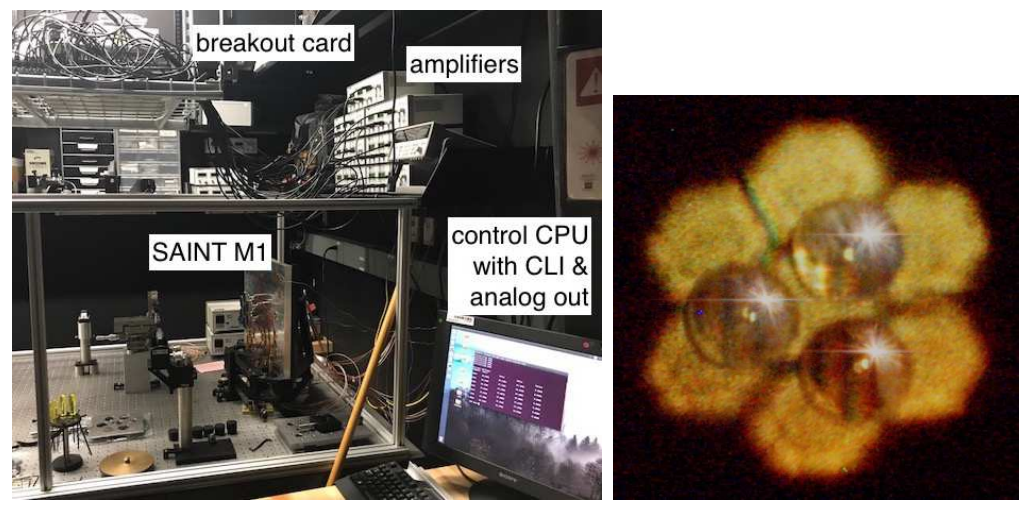

Figure 9. Left: the hardware and controls comprising the SAINT actively-controlled segmented M1, including control computer with a multichannel analog output PCI card connected to a breakout card that relays to the segment control voltage amplifieres. Framework of an air-side enclosure (panels are not shown installed) has been constructed to reduce turbulence and stray light. Right: a lenslet array sampling the vertices of the reimaged SAINT M1 array as the intended hybrid Shack-Hartmann coherent optical edge sensing approach to enabling array alignment. 

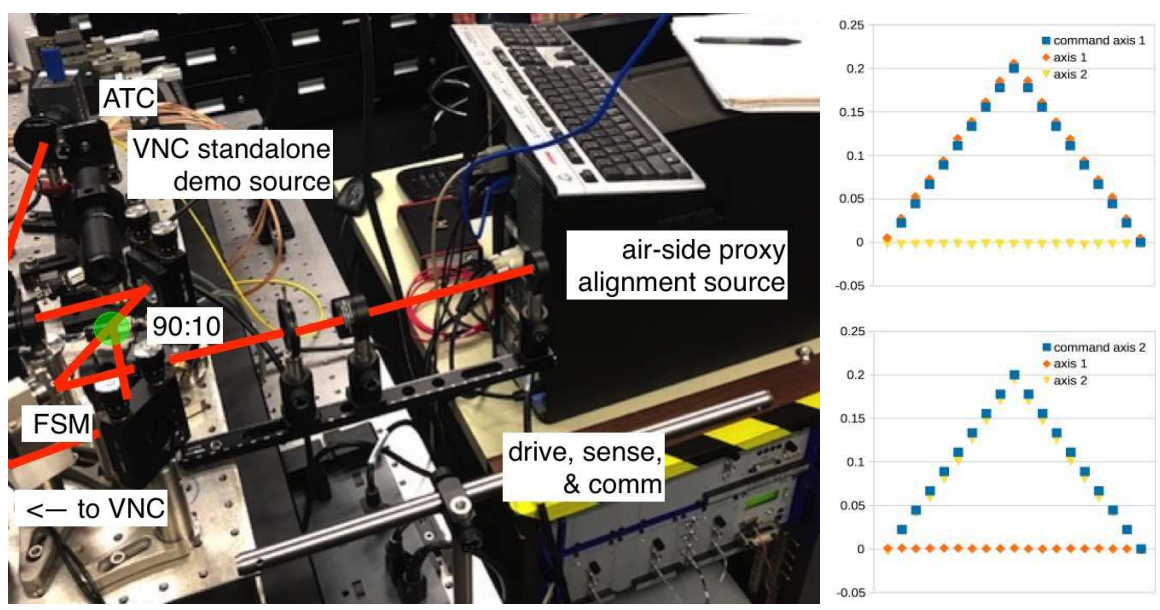

Figure 10. Left: components of the FPS including the key FSM and ATC, as well as relay optics, control electronics, and control CPU. The green circle labeled "90:10" marks the location of a flip-in beamsplitter that reflects $90 \%$ of the light coming from the SAINT air-side optics followed by FSM into the VNC, and transmits $10 \%$ of the light to the ATC. Right: a basic demonstration of FSM open-loop voltage steps commanded, sensed, and passed via the FSM control electronics as commanded by the FPS control CPU.

code has been developed and tested for human-in-the-loop control of the SAINT M1 and is ready for linking with the SAINT M1 WFS for closed-loop control. While it is not necessary, nominally, all three subsystems may eventually be controlled from the same computer.

In an effort to explore a new approach for generating the SAINT end-to-end system segment gap mask (Fig. 11 left), new standalone VNC demonstration segment gap masks (Fig. 11 center right and left) were fabricated to increase each PTT DM segment subaperture throughput by $56 \%$ and add linear span for sensitivity to tip/tilt wavefront error. The new masks use a hole diameter to pitch ratio of $5: 6$, whereas the previous generation masks were 4:6. In addition to improving throughput, the $25 \%$ increase in subaperture diameter improves instantaneous contrast measurement by $\sim 2.5$ times when allowing the core to saturate and using the incoherent sidelobe intensity as a reference (see Fig. 11 center right).

The segment gap mask is shown being aligned to the fully functional Iris AO PTT489 DM in Fig. 12 photos, which also show other new vacuum system additions including electrical filters, a motorized filter wheel, and higher rigidity mounts and plumbing. The gap mask is mounted in a rotation holder that is mounted on a translation stage to set the gap mask position, which is done by observing the subapertures relative to the DM segment gaps and "poking" segments to register centration and clocking. The DM lies in a pupil plane and is conveniently strain-relieved to passive electrical filters designed to attenuate electrical noise at the device's $\sim 2 \mathrm{kHz}$ natural frequency. A camera setup to image the gap mask, which also lies in a pupil plane, replaces an out of plane fold that is installed on a magnetic kinematic mount and folds the beam to the sCMOS high-contast detector, which is rigidly mounted and coupled to copper cooling lines leading to a chiller.
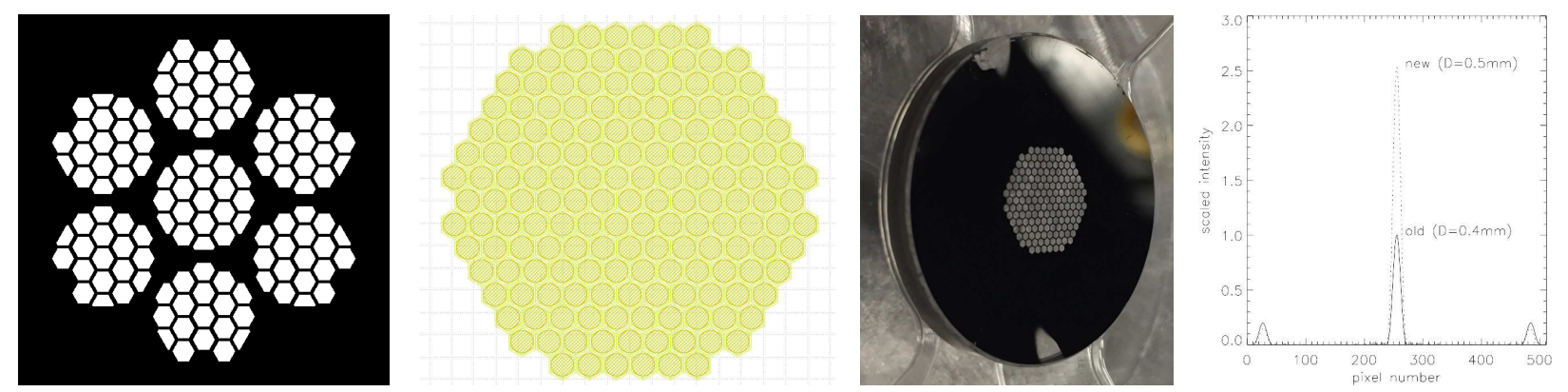

Figure 11. Left to right: the notional SAINT end-to-end mask that is yet to be fabricated, which is defined as the intersection of the clear apertures of the M1 array magnified to the size of an Iris AO PTT489. Segments that span primary mirror gaps will be masked out and not controlled, leaving a total of 127 out of 163 available controllable segments; GDS-II mask rendering for standalone VNC demonstrations showing a hole:pitch ratio of 5:6; a corresponding photo of the first attempt at a transmissive mask fabricated for use in standalone intermediate milestone demonstrations of the VNC with a fully functional PTT489 DM; a cross-section of the theoretical aperture spread functions showing the scaled increase in core to sidelobe peak intensity of the previous generation masks having 4:6 hole:pitch ratio. 

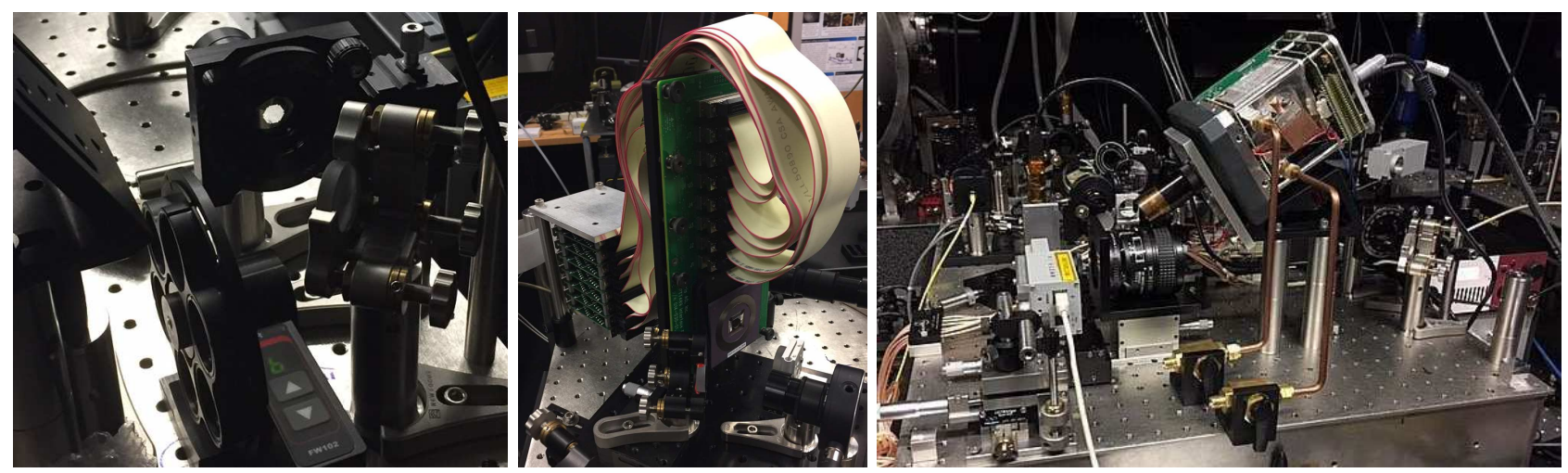

Figure 12. Left: the new higher throughput segment gap mask and motorized filter wheel in the VNC "dark" output. Center: a fully functional Iris AO PTT489 DM strain relieved to passive vacuum electronic filters that mitigate resonances which are otherwise dampened when operating in air. Right: the segment gap mask to DM alignment setup showing a detector in place of the final fold mirror in order to simultaneously observe the location of the mask relative to the DM.

\section{ANCILLARY WORK}

Significant work remains in bringing the SAINT project to successful completion. Multiple sub-efforts described above have been successfully carried out as planned in the project whitepaper. ${ }^{13}$ Additional efforts are being carried out to better ensure satisfying symmetry requirements within the interferometric cavity in order to reach and in the future surpass broadband demonstration milestone goals. The VNC interferometric cavity consists of beamplitter, polarizer (needed to balance field amplitudes), fold mirror, shutter, and achromatic phase shifter pairs, and each element imparts phase and amplitude offsets and asymmetric perturbations to the electric field. It is critical that transmission optics within the VNC cavity have well-matched thicknesses, parallelism, antireflection coatings, and alignment, lest bulk dispersion and surface interface phase effects severely compromise performance. Hybridizing components, where possible, has the potential to 1) improve the contrast floor, 2) simplify the alignment effort, 3) make the layout more compact and stable. We are exploring the design of hybrid non-beamsplitters/combiners and polarizers shown schematically in Fig. 13 to mitigate chromatic shear associated with small, but significant ill-effects of refraction stemming from residual thickness and wedge errors in these components, as well as asymmetry in their alignment.

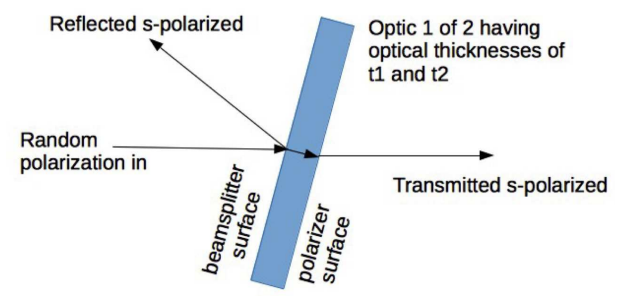

Figure 13. A hybrid beamplitter and polarizer concept being explored to improve broadband symmetry. Hybridizing these two pairs of components to a single pair has the potential for mitigating ill-effects of dispersion associated with unbalanced thickness, parallelism, and refraction errors limited by fabrication techinques and metrology precision.

Beyond and also relating to broadband performance, reaching higher contrasts over a greater coronagraphic image plane discovery space with various coronagraphic approaches is, in theory, better enabled by complex wavefront control of polishing and alignment errors giving rise to a need for phase control, as well as amplitude control to address transmission non-uniformity. The notion of achieving complex wavefront control by pairing a PTT DM with a passive waveguide array optical element (rather than two deformable mirrors) is an aging idea with few reported test results. In theory, the idea of using a single-mode fiber array (SFA) not only affords amplitude control, but also filters low spatial frequency errors in individual DM segments.

With SFA units on-hand for testing, we have designed and built an experimental setup to simultaneously observe images and pupils produced by these units as compared to what is observed using the segment gap masks used in VNC demonstrations. The raytrace and breadboard setup are shown in Fig. 14. The setup includes multiple delay lines to allow for coherent 4-beam white light interference and quantify residual speckle at different corresponding spatial frequencies. Software was written to control and capture frames from the 

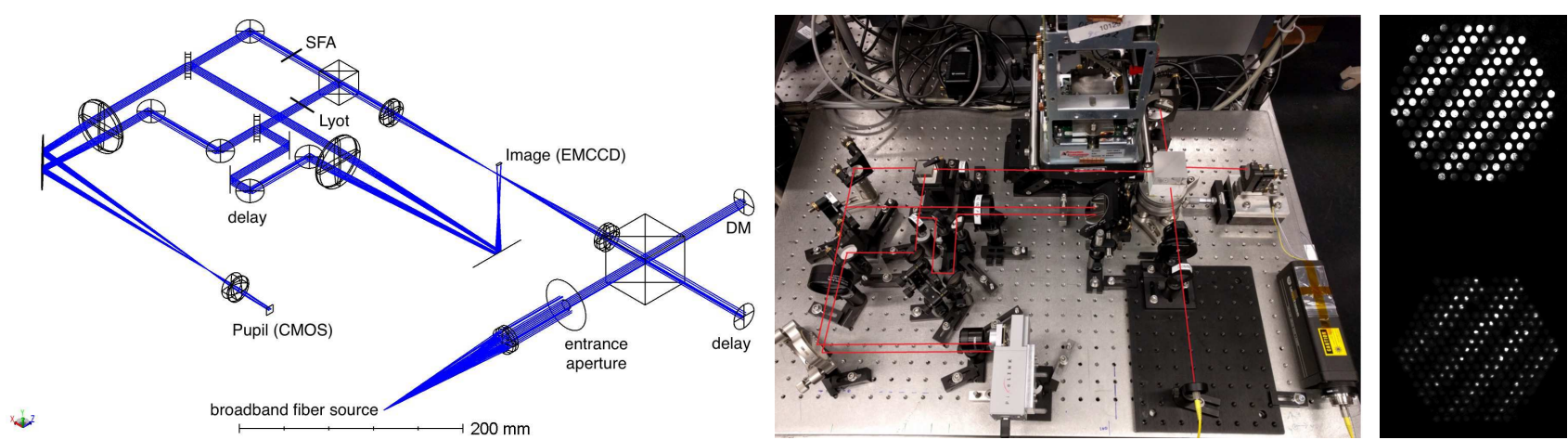

Figure 14. From left to right: the SFA testbed raytrace, breadboard, and initial pupil images observed through the segment gap mask (top) and SFA (bottom).

detectors. It is noted that optics following the spatial filter arrays should be superpolished to not corrupt the measurement, a potential shortcoming of the setup that could be remedied through other means. A low-noise, high well-depth detector is being used for the imaging detector to enable higher contrast measurement of the speckle floor. Initial results with the SFA as observed on the image plane detector are poor, and further testing and analysis will be required to determine if it is due to the quality of the SFA array or the manner in which it is being used. A segmented PTT DM remains to be integrated with the testbed along with software to demonstrate the key benefity of amplitude control authority through the SFA.

\section{SUMMARY}

SAINT aims to advance coronagraphic techniques for future segmented aperture telescopes. SAINT integrates the VNC with an actively-controlled hexagonal array segmented mirror telescope via a FPS. A successful SAINT effort will culminate in a demonstration of high-contrast imaging with a complex aperture enabled by sequential wavefront control. This SAT/TDEM-funded work specifically targets achieving a contrast of $10^{-8}$ over a $1 \lambda / D$ diameter circular area centered at $4 \lambda / D$ using a filtered source spectral bandpass of $20 \mathrm{~nm}$ centered on $633 \mathrm{~nm}$. A stretch goal will be to achieve $10^{-9}$ contrast at a smaller IWA, possibly $\sim 3 \lambda / D$, and a broader bandpass of $40 \mathrm{~nm}$. Achieving this will further advance coronagraphy towards being adaptable to segmented aperture telescopes. Prior to attempting the end-to-end Milestone \#3 goal, narrowband performance will need to be repeated and broadband high-contrast performance demonstrated for the first time with the VNC operating inside its vacuum chamber, independent of the air-side actively-controlled segmented telescope optics. ${ }^{13}$

The milestones are taken to represent feasible objectives based on current VNC Technology Readiness Levels (TRLs), and the TRL of component technologies, including wavefront sensing and control (WFS/C) with a segmented aperture telescope, DM, FPS, and APS. The SAINT project takes an important step towards enabling exoplanet coronagraphy for segmented apertures and ultimately for future missions such as a Large UV/Optical/Infrared (LUVOIR) telescope ${ }^{14}$ or the Habitable Exoplanet Imager (HabEx). ${ }^{15}$ Upon completion of this effort, sub-system technology will be properly positioned to address the additional steps required to mature this high-contrast imaging approach to TRL 6 . The majority of the requisite hardware and software integration has been completed, and the demonstration phase of the project should commence imminently with results presented in forthcoming reports and publications.

\section{ACKNOWLEDGMENTS}

The authors acknowledge support from the NASA/GSFC Internal Research and Development (IRAD) and NASA Strategic Astrophysics Technology (SAT) Technology Development for Exoplanet Missions (TDEM) programs, as well as the NASA/GSFC Education Office. 


\section{REFERENCES}

1. R. G. Lyon, M. Clampin, P. Petrone, U. Mallik, T. Madison, and M. R. Bolcar, "High contrast vacuum nuller testbed (VNT) contrast, performance, and null control," Proc. SPIE 8442, Sept. 2012.

2. M. Clampin, R. Lyon, P. Petrone III, U. Mallik, M. Bolcar, T. Madison, and M. Helmbrecht, "Visible nulling coronagraph technology maturation: High contrast imaging and characterization of exoplanets," tech. rep., NASA/Technology Development for Exoplanet Missions Final Report, JPL Document D-80950, https://exep.jpl.nasa.gov/technology/Clampin_Report_FINAL.pdf, 2013.

3. O. Guyon, E. A. Pluzhnik, M. J. Kuchner, B. Collins, and S. T. Ridgway, "Theoretical Limits on Extrasolar Terrestrial Planet Detection with Coronagraphs," Astrophys. J. Sup. 167, pp. 81-99, Nov. 2006.

4. E. Serabyn, "Nulling interferometry: symmetry requirements and experimental results," Proc. SPIE 4006, pp. 328-339, July 2000.

5. B. A. Hicks, "Exoplanet detection and characterization via parallel broadband nulling coronagraphy," J. Ast. Tel. Inst. \& Sys. 2, p. 011015, Jan. 2016.

6. E. Bolmont, A.-S. Libert, J. Leconte, and F. Selsis, "Habitability of planets on eccentric orbits: Limits of the mean flux approximation," Aston. \&S Astrophys. 591, p. A106, June 2016.

7. C. C. Stark, A. Roberge, A. Mandell, and T. D. Robinson, "Maximizing the ExoEarth Candidate Yield from a Future Direct Imaging Mission," Astrophys. J. 795, p. 122, Nov. 2014.

8. B. Hicks, R. Lyon, M. Bolcar, M. Clampin, and P. Petrone, "High-contrast visible nulling coronagraph for segmented and arbitrary telescope apertures," Proc. SPIE 9143, 2014.

9. B. A. Hicks, R. G. Lyon, P. Petrone, I. Miller, M. R. Bolcar, M. Clampin, , M. Helmbrecht, and U. Mallik, "Demonstrating broadband billion-to-one contrast with the Visible Nulling Coronagraph," Proc. SPIE 9605, 2015.

10. B. A. Hicks, R. G. Lyon, P. Petrone, I. Miller, M. R. Bolcar, M. Clampin, , M. Helmbrecht, and U. Mallik, "Recent developments with the Visible Nlling Coronagraph," Proc. SPIE 9907, 2016.

11. B. Hicks, M. Bolcar, R. Lyon, M. Clampin, T. Madison, U. Mallik, P. Petrone III, and M. Helmbrecht, "Technology milestone \#2 final report: Achromatic visible nulling coronagraph technology maturation," tech. rep., NASA/Technology Development for Exoplanet Missions, JPL Document D-1547413, https://exoplanets.nasa.gov/exep/technology/TDEM-awards/, 2016.

12. B. A. Hicks, R. G. Lyon, P. Petrone, M. Ballard, M. R. Bolcar, J. Bolognese, M. Clampin, P. Dogoda, D. Dworzanski, M. A. Helmbrecht, C. Koca, and R. Shiri, "The Segmented Aperture Interferometric Nulling Testbed (SAINT) I: overview and air-side system description," Proc. SPIE 9904, p. 990420, July 2016.

13. B. Hicks, M. Bolcar, P. Petrone III, and M. Helmbrecht, "Technology milestone \#3 whitepaper: Nulling of an actively-controlled segmented aperture telescope," tech. rep., NASA/Technology Development for Exoplanet Missions, JPL Document D-1549543, https://exoplanets.nasa.gov/exep/technology/TDEM-awards/, 2016.

14. M. R. Bolcar, K. Balasubramanian, J. Crooke, L. Feinberg, M. Quijada, B. Rauscher, D. Redding, N. Rioux, S. Shaklan, H. P. Stahl, C. Stahle, and H. Thronson, "Technology gap assessment for a future large-aperture ultraviolet-optical-infrared space telescope," J. Ast. Tel. Inst. ES Sys. 2, p. 041209, 2016.

15. B. Mennesson, S. Gaudi, S. Seager, K. Cahoy, S. Domagal-Goldman, L. Feinberg, O. Guyon, J. Kasdin, C. Marois, D. Mawet, M. Tamura, D. Mouillet, T. Prusti, A. Quirrenbach, T. Robinson, L. Rogers, P. Scowen, R. Somerville, K. Stapelfeldt, D. Stern, M. Still, M. Turnbull, J. Booth, A. Kiessling, G. Kuan, and K. Warfield, "The Habitable Exoplanet (HabEx) Imaging Mission: preliminary science drivers and technical requirements," Proc. SPIE 9904, p. 99040L, July 2016. 